\title{
Efficacy and safety of tocilizumab in a real-life observational cohort of patients with polyarticular juvenile idiopathic arthritis
}

Minna-Maija Grönlund ${ }^{1}$, Terhi Remes-Pakarinen², Liisa Kröger ${ }^{2}$, Kati Markula-Patjas ${ }^{3}$, Maria Backström ${ }^{4}$, Anne Putto-Laurila ${ }^{1}$, Kristiina Aalto ${ }^{5}$, Paula Vähäsalo ${ }^{6}$

1) Department of Pediatrics, Turku University Hospital, Turku, Finland Correspondence to: Minna-Maija Grönlund, Department of Pediatrics, Turku University Hospital, Kiinamyllynkatu 4-8, 20520 Turku, Finland.

E-mail: minna-maija.gronlund@tyks.fi

2) Department of Pediatrics, Kuopio University Hospital, Kuopio, Finland

3) Department of Paediatrics, Tampere University Hospital, Tampere, Finland University of Tampere, Tampere, Finland

4) Department of Pediatrics, Vaasa Central Hospital, Vaasa, Finland

5) Department of Children and Adolescents, Helsinki University Hospital, Helsinki, Finland Pediatric Research Center, University of Helsinki, Helsinki, Finland

6) Medical Research Center Oulu, Oulu University Hospital and University of Oulu, Oulu, Finland Department of Children and Adolescents, Oulu University Hospital, Oulu, Finland PEDEGO Research Unit, University of Oulu, Oulu, Finland

\section{Abstract}

Objectives: To evaluate the patterns of usage, efficacy and safety of tocilizumab in polyarticular JIA.

Methods: An observational study of 56 consecutive polyarticular JIA patients was conducted using patient charts and electronic JIA databases. Efficacy was assessed by tocilizumab survival, rates of low disease activity (LDA) and of inactive disease by 10-joint Juvenile Arthritis Disease Activity Score (JADAS-10), and of clinically inactive disease according to Wallace's preliminary criteria. Efficacy and rate of adverse events (AEs) were evaluated during a 24-month period after tocilizumab commencement.

Results: Tocilizumab was started on average as third-line biological agent (median, range first- to fourth-line) at a median disease duration of 5.2 years (interquartile range 3.0-7.7). Survival rates were $82 \%$ at 12 months and $64 \%$ at 24 months. The reasons for discontinuation were inadequate treatment effect in $50 \%$, AE plus inadequate treatment effect in $37.5 \%$ and $A E$ alone in $12.5 \%$. LDA (JADAS-10 $\leqslant 3.9$ ) was reached in $58 \%$ at 12 months and in $84 \%$ at 24 months, inactive disease (JADAS-10 $\leqslant 0.7$ ) in $19 \%$ and $44 \%$, and clinically inactive disease in $28 \%$ and $46 \%$, respectively. The rate of AEs was 200.9/100 patient years and of serious AEs 12.9/100 patient years.

Conclusion: Survival of tocilizumab was high and a large proportion of the treatment-resistant patients reached LDA at 12 months of treatment. The LDA rate continued to increase throughout 24 months. The rates of AEs and serious AEs were higher than in register studies but lower than in the originator study of tocilizumab.

Keywords: juvenile idiopathic arthritis, biological therapy, tocilizumab, JADAS, inactive disease, adverse event

Topic: arthritis, juvenile rheumatoid, safety, tocilizumab, adverse event, disease remission

Key messages

-Tocilizumab survival was $82 \%$ at 12 months in polyarticular JIA patients with treatment-resistant disease.

-A large proportion of the patients reached low disease activity at 12 months. 
-The rates of adverse events and serious adverse events were higher than in previous register studies.

\section{Introduction}

JIA is a heterogeneous collection of diseases categorized into seven subtypes according to the ILAR classification criteria [1]. The majority of children with different subtypes develop a so-called polyarticular course of the disease with inflammation in five or more joints [2,3]. Children with polyarticular JIA (pJIA) have a more treatment-resistant disease than those with fewer joints affected, and they have longer periods of active disease [3-5], which is associated with higher risk of joint damage [6]. Accordingly, recent treatment recommendations for pJIA suggest starting biological DMARDs (bDMARDs) as initial treatment or after 3-6 months' use of synthetic DMARDs (sDMARDs) with non-response [7-9]. A trend of using bDMARDs earlier in the disease course and in JIA patients with lower disease activity has been detected in several studies [5, 10, 11].

Currently approved biological agents for the treatment of pJIA include abatacept, adalimumab, etanercept and tocilizumab (both European Medicines Agency, and Food and Drug Administration) and golimumab (European Medicines Agency). First-line biological agents in the treatment of pJIA have mostly been TNF inhibitors, partly due to the order of approval [11,12] and national regulatory rules [11].

Tocilizumab, a humanized monoclonal IL-6 receptor antibody, was approved for treatment of pJIA in 2013 as first-line biological agent in the case of inadequate response to MTX

(https://www.ema.europa.eu/medicines/human/EPAR/roactemra). According to the German BiKeR study, during 2011-15 tocilizumab was used as first-line biological agent in $18.9 \%$ of pJIA patients ever using tocilizumab [12]. Horneff et al. [12] found no difference in the efficacy of adalimumab, etanercept or tocilizumab either as first-line or second-line biological agent in pJIA. Similarly, a metaanalysis of the original withdrawal studies of abatacept, adalimumab, etanercept and tozilicumab in pJIA patients found no difference between these bDMARDs in efficacy of preventing flares or in improving symptoms [13].

Randomized controlled trials remain the gold standard for establishing the efficacy and short-term safety of new therapeutic agents. However, such studies have strict criteria of patient selection with regard to disease duration and disease severity; in addition, the withdrawal design used in the JIA biological agent studies can overestimate the effect of the trial agent [14, 15]. Thus, the results of randomized controlled trials are hard to generalize to real-life clinical practice. Consequently, observational data of unselected real-life patients provides important additional information to complement the results of randomized controlled trials.

Today, there is only limited information from observational studies investigating the use of tocilizumab in JIA patients $[11,12,16]$. We conducted a nationwide observational study in Finland to detect the patterns of usage, efficacy and safety of tocilizumab in pJIA patients in routine clinical practice.

\section{Methods}

\section{Study design and patients}

All consecutive pJIA patients aged 2-17 years attending one of the existing five tertiary university hospitals in Finland or one of the secondary central hospitals (Vaasa Central Hospital) for whom the treating physician had decided to commence tocilizumab after July 2013 were included in a multicentre retrospective chart review. In addition, the electronic JIA databases of each hospital were used to collect the JIA core outcome variables (see below). Since tocilizumab was a new type of biologic treatment for pJIA it is very unlikely that many pJIA patients would have been treated in the other secondary central hospitals not included in this study.

This study yielded 62 JIA patients with seropositive or seronegative polyarthritis or extended oligoarthritis (i.e. pJIA) according to the ILAR classification criteria [1]. Patients had started i.v. tocilizumab between October 2013 and September 2017. Only patients starting tocilizumab for the 
first time according to the official dose instructions (dose $8 \mathrm{mg} / \mathrm{kg}$ every 4 weeks in patients weighing $\geqslant 30 \mathrm{~kg}$ and $10 \mathrm{mg} / \mathrm{kg}$ every 4 weeks in patients weighing $<30 \mathrm{~kg}$ ) were included. The dose could be rounded to the closest practical amount but no more than $15 \%$ deviation from the official dose was allowed. Six patients were excluded from the study because of the dose deviation, leaving a final study population of 56 patients.

\section{Assessment and outcome parameters}

Data at clinical visits closest to $0,3,6,12,18$ and 24 months after starting tocilizumab were included. The follow-up ended at the last available visit (patients continuing tocilizumab) or the last recorded visit closest to the discontinuation of tocilizumab. Median follow-up was 23.6 months [interquartile range (IQR) 19.0-25.3] for those continuing the treatment and 6.7 months (IQR 5.8-16.8) for those who discontinued the treatment.

Demographic and clinical characteristics of the patients collected at initiation of tocilizumab treatment included: age at diagnosis, disease duration, JIA category, prevalence of anterior uveitis and previous treatment of JIA (sDMARDs, bDMARDs, systemic and intra-articular glucocorticoids).

Treatment patterns of tocilizumab (dose and dose interval, reasons for dose modifications, proportion of patients discontinuing tocilizumab and reasons for discontinuing, proportion of patients on tocilizumab monotherapy at entry and during follow-up), concomitant sDMARD therapy and use of intra-articular glucocorticoids were recorded.

The JIA core outcome variables (number of joints with active arthritis, joints with limited range of motion, ESR, CRP, physician's global assessment of disease activity and patient's/parent's global assessment of well-being, both on 100-mm visual analogue scale (VAS), the Childhood Health Assessment Questionnaire-Disability Index (CHAQ-DI), range 0-3, 0 = no disability) were recorded.

Efficacy of the treatment was evaluated by drug survival and 10-joint Juvenile Arthritis Disease Activity Score (JADAS-10) [17]. The new Finnish JADAS-10 cut-off values of low disease activity (LDA) $(\leqslant 3.9)$ and inactive disease $(\leqslant 0.7)$ were used [18]. In addition, the number of patients with clinically inactive disease was determined by Wallace's preliminary criteria, since they take into account the activity of uveitis [19].

Assessment of safety included rates of adverse events (AEs) and serious adverse events (SAEs). AEs are defined as any untoward medical occurrence in a patient administered a pharmaceutical product, even without a causal relationship with the treatment. SAEs are defined as events that are fatal or immediately life-threatening, or require inpatient care or prolong hospitalization, or result in persistent or significant disability/incapacity, congenital anomaly, or spontaneous or elective abortion, or require medical or surgical intervention to prevent a serious outcome. Rates of abnormal laboratory results in total blood count, neutrophil count, transaminases, cholesterol, low-density lipoprotein, high-density lipoprotein and triglyceride values found in routine laboratory tests were recorded. Abnormal laboratory results were defined as values leading to adjustment in tocilizumab treatment or concomitant medication. The cut-off values for neutrophils and transaminases (alanine aminotransferase) were $\leqslant 1.0 \times 109 / \mathrm{l}$ and $\geqslant 70-100 \mathrm{U} / \mathrm{l}$, respectively. Injuries and flares of JIA were not reported as AEs. AEs were coded according to the Medical Dictionary for Regulatory Activities, version 21.1 (https: / /www.meddra.org).

\section{Ethics}

The parents were given verbal and written information about the study and they provided written informed consent. Permission for the study was received from the Ethics Committee of the Hospital District of South-West Finland and the ethical review board of the paediatric departments of each hospital.

\section{Statistics}

Data are descriptive and mostly summary statistics are presented as mean and s.d., median and range, or IQR as relevant. The Mann-Whitney $U$ test was used to compare JADAS values in patients with monotherapy and combination therapy at 12 months of follow-up. The comparison was done only between those patients who had been on monotherapy or combination therapy for the whole 12- 
month period. Friedman's test was used to analyse the time effect between all of the follow-up points on continuous variables and Cochrane's Q test for categorical variables. No post hoc analysis was done. SPSS version 25 (IBM, Armonk, NY, USA) was used for the analysis.

The numbers of patients included at different time points were: commencement of tocilizumab, 56 patients; 3 months, 54 patients ( 2 patients had no clinical visit); 6 months, 55 patients ( 1 patient discontinued earlier); 12 months, 47 patients ( 9 discontinued earlier); 18 months, 39 patients (10 discontinued earlier, 7 followed up for shorter duration); and 24 months, 29 patients (16 discontinued earlier, 11 followed up for shorter duration). In addition, due to missing information on core set variables, JADAS was missing in three to eight patients per visit (8-15\% of patients included at each time point). We compared the baseline characteristics included in Table 1 between patients with complete JADAS values $(n=43)$ and those with some missing JADAS values at one or more clinical visits $(n=13)$ using the Mann-Whitney $U$ test and $x 2$ test or Fisher's Exact test, as relevant. No statistically significant differences were found in the baseline characteristics between these two groups.

\section{Results}

Fifty-six pJIA patients were included in the study. The baseline patient and disease characteristics are shown in Table 1. Tocilizumab was the first-line biologic in six patients (11\%) and these treatments were started in the years 2015-17. In patients receiving biologic therapy before tocilizumab, the median number of previous biologics was 2 (range 1-4). The distribution of different biologics used is shown in Fig. 1A. The reasons for switching biological agents are shown in Fig. 1B. Before starting tocilizumab all patients had used sDMARDs; all had used MTX, and the median number of different sDMARDs used was 2.5 (range 1-5, including prednisolone and sDMARDs in different combinations). The concomitant sDMARD treatment at tocilizumab commencement is shown in Table 1.

Tocilizumab modifications, survival and discontinuations

The tocilizumab infusion interval was shortened in nine patients due to inefficacy: in one patient to every other week and in eight patients to every 3 weeks. After the shortened infusion interval seven patients had at least one follow-up visit (6-12 months after the change): six patients continued tocilizumab up to the last available clinical visit and one discontinued tocilizumab 1 month after the change due to inefficacy. The median JADAS-10 score decreased from 9.3 (IQR 7-10.8) to 5.8 (IQR 2.78.3) (from the clinical visit before the change to the last clinical visit after the change) in those continuing tocilizumab treatment. One patient had infusions every 5 weeks due to frequent neutropenia.

Drug survival at 6 months, 1 and 2 years of treatment was 91, 82 and 64\%, respectively (Fig. 2). Altogether 16 patients discontinued tocilizumab at the median treatment duration of 6.0 months (range 2.8-20.9 months). The reasons for discontinuation are given in Table 2. For those who discontinued the tocilizumab treatment the median JADAS-10 was 5.7 (IQR 2.3-9.7), the median number of active joints 1.5 (IQR 0-4.0), the median CHAQ-DI 0.625 (IQR 0.1875-1.0) and the median patient's/parent's pain-VAS $23.5 \mathrm{~mm}$ (IQR 5-47 mm) at the last visit.

\section{Tocilizumab monotherapy}

Nine patients (16\%) started tocilizumab treatment as monotherapy and during the follow-up 11 more patients switched to monotherapy. Only one patient with monotherapy restarted sDMARDs at 24 months. The proportion of monotherapy was $28 \%$ at 12 months and $38 \%$ at 24 months.

The median JADAS-10 scores in the patients with continuous monotherapy from the start of tocilizumab $(n=8)$ and those with continuous combination therapy (tocilizumab and sDMARD) $(n=31)$ were comparable at 12 months of follow-up $(P=0.5)$ (data not shown).

\section{Tocilizumab efficacy}

The proportions of patients with LDA (JADAS-10 $\leqslant 3.9$ ), inactive disease (JADAS-10 $\leqslant 0.7$ ) and those with clinically inactive disease according to Wallace's preliminary criteria are shown in Fig. 3 . The median JADAS-10 decreased from 7.6 (IQR 5.4-12.1) at commencement to 1.2 (IQR 0-2.5) at 24 months 
( $P<0.0001$ Friedman's test for difference over time) and the median number of sDMARDs per patient from 2.5 (IQR 2-3) to 1.0 (IQR 0-1) ( $<0.0001$ Friedman's test). The proportion of patients receiving intra-articular glucocorticoid injections decreased from $89 \%$ at baseline (i.e. injections within 6 months prior to tocilizumab start) to $17 \%$ at 24 months (i.e. injections between the 12- and 24 -month clinical visits) ( $P=0.004$ Cochran's $Q$ test for difference over time). The proportion of patients receiving systemic glucocorticoids at the clinical visit decreased from $27 \%$ at baseline to $17 \%$ at 24 months of follow-up ( $P<0.0001$ Cochran's $Q$ test). There was a clear decrease in the patient's/parent's pain-VAS from median 28 (IQR 11-51) to 1.5 (IQR 0-40) ( $P=0.032$ Friedman's test) and CHAQ-DI from 0.625 (IQR 0.125-1.0) to 0.0625 (IQR 0-0.5) from baseline to 24 months $(P=0.0001$ Friedman's test), respectively.

\section{Uveitis}

Eleven patients had a previous history of uveitis at tocilizumab commencement. Five of them had active uveitis when starting tocilizumab; three continued to have active uveitis throughout the tocilizumab treatment (two continued tocilizumab for 24 months and one discontinued at 21 months because of active uveitis and arthritis), one had a single flare and one had no flares during the followup. In two patients with no uveitis at the commencement of tocilizumab a uveitis flare was observed at the last visit (18 and 24 months, respectively) and the other four patients had no flares during the follow-up. No new-onset uveitis occurred during the tocilizumab treatment.

\section{Adverse events}

A total of 85.1 patient years (PYs) was captured. Forty patients (71\%) experienced a total of 171 AEs (Table 3). The rate of AEs was 200.9/100 PYs. Infections were the most common AEs (54 infections), detected in 24 patients (43\%). The most common infections are shown in Table 3; other infections included outpatient pneumonia, sinusitis, conjunctivitis and verrucae (one of each). Gastrointestinal AEs were also frequent [31 AEs in 18 patients (32\%)].

Neutropenia was reported 22 times (25.9/100 PYs) in 10 patients (18\%) and elevated transaminases 11 times (12.9/100 PYs) in 8 patients (14\%). Six patients had recurrent neutropenia and two patients had recurrent elevation of transaminases. One patient had elevated cholesterol values (total cholesterol $5.9 \mathrm{mmol} / \mathrm{l}$, low-density lipoprotein $4.2 \mathrm{mmol} / \mathrm{l}$ ) and tocilizumab was discontinued because of this and inefficacy.

Three infusion reactions occurred, all during the first tocilizumab infusion and none leading to discontinuation. One patient with shivering, fever, flush and nausea, required overnight hospitalization with i.v. glucocorticoids and hydration. This patient continued tocilizumab treatment for the entire follow-up (26 months) but was given antihistamine medication and acetaminophen before infusions. The other two patients had only skin reactions (undefined rash and urticaria, respectively).

The total number of SAEs was 11 (Table 3), occurring in nine patients (16\%). The rate of SAEs was 12.9/100 PYs. Three cases of infectious SAEs were recorded; influenza A infection with concomitant bacterial sepsis, Bartholin's gland abscess and tooth infection, with both of the latter requiring surgical treatment. No cases of mycobacterial infection, death, malignancy, new onset of autoimmunity or pregnancy were reported.

\section{Discussion}

The current recommendations suggest TNF inhibitors as the first choice of treatment in children with pJIA if sDMARDs have failed; thereafter another TNF inhibitor or abatacept is recommended for the second-line biological treatment [7]. However, the evaluation underpinning these recommendations did not include studies of tocilizumab for pJIA as no such studies were available at that time. Recent German pJIA therapy algorithms include tocilizumab in the first-line use of biological agents [9], as do the Childhood Arthritis and Rheumatology Research Alliance (CARRA) Consensus Treatment Plans [8]. The pathways of using biological agents in clinical practice are constantly changing [10, 20] and vary considerably in different countries due to local regulations and availability of the biological agents $[11,12,20-22]$. Since there is rather short experience of tocilizumab in the treatment of pJIA, only 
few register and observational reports have been published on the safety and efficacy of tocilizumab so $\operatorname{far}[11,12,16]$.

This study evaluated a nationwide cohort of 56 consecutive Finnish pJIA patients starting tocilizumab treatment between 2013 and 2017. Tocilizumab was on average a third-line biological treatment, the median disease duration was 5.2 years and the median JADAS-10 was 7.6 at tocilizumab

commencement, depicting a cohort with treatment-resistant polyarthritis. Taking this into account, drug survival at 12 months (82\%) was high. It was higher than reported on TNF inhibitors as second-line or third-line biological therapy at 12 months in non-systemic JIA (50-54\%) [23]. A higher survival of tocilizumab compared with TNF inhibitors was also detected in the BiKeR registry, a study that included patients in exactly the same JIA categories as ours and mostly using tocilizumab as at least second-line biological agent [12]. Nevertheless, a steady decline in the survival rate of tocilizumab was noted throughout the follow-up in our study, ending at $64 \%$ at 24 months. Tocilizumab was discontinued solely due to inadequate treatment effect in half of the cases, whereas AEs were associated with discontinuation in the other half. Horneff et al. [12] reported discontinuation of tocilizumab due to inefficacy in $39 \%$ and intolerance in $9 \%$ of the cases, while in $43 \%$ of the cases the reason was not specified. In the study by Otten et al. [23] a second-line biological agent was discontinued due to inefficacy in $64 \%$ and to $A E$ in $23 \%$ of the cases; however, these results are not readily comparable to ours since their study included $50 \%$ of patients with systemic JIA.

It is widely recognized that SDMARDs are not well tolerated in JIA patients [24]. Accordingly, the rate of tocilizumab monotherapy increased in this study from $16 \%$ at tocilizumab commencement to $38 \%$ at 24 months. The rate of tocilizumab monotherapy in our study was still lower than the $54 \%$ rate found in the BiKeR registry [12]. In the original placebo-controlled withdrawal study of 188 pJIA patients (the CHERISH study) the response rates were higher in the group of JIA patients receiving a combination of tocilizumab and MTX compared with tocilizumab monotherapy. However, tocilizumab was very efficacious even as monotherapy; at 40 weeks the ACRpedi70 response was achieved in $67 \%$ and $53 \%$ of patients with and without MTX, respectively [25]. Conversely, we found no difference in the efficacy of tocilizumab (assessed by JADAS-10 scores) at 12 months of follow-up between patients with monotherapy and those with tocilizumab and SDMARD combination. Still, this study may have been too small to discover subgroup differences. Interestingly, it was found in the BiKeR registry that the survival rate of biological treatment (etanercept, adalimumab and tocilizumab) at 5 years' followup was the same in pJIA patients with biological monotherapy and with MTX combination therapy [12].

According to the recent recommendations on the treatment goal of JIA, LDA is considered a valid target for patients with longstanding, persistently active pJIA [26]. This resembles the disease situation in our patients at tocilizumab commencement. There are only few studies reporting treatment outcomes for this kind of patient. Using the recent Finnish JADAS-10 cut-off values [18] a large proportion of the patients remaining on tocilizumab reached LDA at 12 months (58\%) and 24 months (84\%). If the cut-off values of Consolaro et al. [27] are used, the treatment responses are the same or somewhat higher in our cohort (data not shown) compared with the pJIA patients in the BiKeR registry using tocilizumab; at 12 months LDA was reached in $58 \%$ in our cohort vs $61 \%$ in the BiKeR study and remission in 28 vs $21 \%$, respectively, and at 24 months LDA in 84 vs $62 \%$ and remission in 48 vs $23 \%$, respectively [12]. Clinically inactive disease at 12 months was reached in 19\% (by JADAS-10 $\leqslant 0.7$ ) to $28 \%$ (by Wallace's preliminary criteria) of our patients, which is higher than that previously reported in patients switching biological agents $(7-17 \%)[23,28]$. The improvement in the clinical outcome of our study patients was also reflected in the decreased usage of different sDMARDs, systemic and intra-articular glucocorticoids, and in the lower pain-VAS.

We found a higher rate of SAEs (12.9/100 PYs) for tocilizumab than that reported in a recent European retrospective cohort of paediatric patients with inflammatory rheumatic diseases $(8.2 / 100 \mathrm{PYs}$, total exposure 245 PYs) [29] and that of pJIA patients in the BiKeR registry (4.1/100 PYs, total exposure 72.5 PYs) [12]. The largest observational safety report on tocilizumab in JIA has been that of 435 PYs from the BiKeR registry, where the rate of SAEs was 17.3/100 PYs [16]. However, this congress abstract probably also includes patients with systemic JIA, which would explain the high number of SAEs $[16,30]$. Nevertheless, the rate of SAEs in our study was comparable to that of another retrospective observational cohort of Finnish JIA patients treated with biological agents (11.4/100 PYs), of whom 39\% were biological agent switchers [31]. As pointed out by Tarkiainen and colleagues, data obtained from patient charts seem to be a more reliable source of information on AEs than register data [31]. This may be due to a combination of more frequent data collection available from all hospital visits and the public health care system in Finland, where all visits that require hospital 
treatment are very likely to be handled by the same tertiary hospital taking care of these JIA patients. Actually, the rate of SAEs in our study was comparable to the CHERISH study (12.5/100 PYs), but the rate of AEs was clearly lower than in CHERISH (480/100 PYs) [25]. The extended safety data of 2 years' follow-up in the CHERISH study found no change in the safety profile of tocilizumab [32], neither did the extension data of Polish and Russian JIA patients included in the CHERISH study [33]. Previous studies on tocilizumab safety in European JIA patients have not reported any cases of malignancies, tuberculosis, demyelinating disorders or deaths [12, 25, 33], which was also the case in our study. One exception to this has been published recently by Horneff et al. [16], who reported one malignancy.

It could be hypothesized that the use of multiple, consecutive biological agents could have caused the higher rate of SAEs and AEs in this study. However, previous reports on JIA do not support this notion. No difference was found in the rate of AEs or SAEs between the JIA patients on first-line biological agents and those switching biological agents in the BiKeR registry; in contrast, more infectious AEs were found in the first-line biologic group compared with the biologic-switcher group [28]. In addition, the rate of AEs and SAEs were comparable during the first to the third course of biological agents in JIA patients of the Dutch ABC Register [23].

The rate of specific AEs is difficult to compare between studies of biological treatments due to the differences in the classification of AEs and in the type of the study (i.e. a single-centre, registry or placebo-controlled study, or a long-term extension cohort) [29, 34]. Furthermore, the rates of AEs associated with tocilizumab differ depending on the indication (i.e. systemic JIA or pJIA) [25, 30, 35]. However, one typical AE for tocilizumab is neutropenia [35]. We detected a higher rate of grade 3 neutropenia $(\leqslant 1.0 \times 109 / \mathrm{l})$ in $18 \%$ of our patients compared with $5.9 \%$ reported in the CHERISH study patients during 2 years of follow-up [35]. Pardeo and colleagues found no association between development of neutropenia and tocilizumab trough levels or concomitant MTX use, and neutropenia was not associated with the development of infections [35]. The difference in the rate of neutropenia might be explained by the fact that many of our patients used concomitant sDMARDs other than MTX as well as combinations of sDMARDs, while only MTX and glucocorticoids were allowed in the CHERISH study [25]. sDMARDs other than MTX during biological treatment have been associated with higher rates of AEs and SAEs [29].

No new-onset uveitis was found in our patients during tocilizumab treatment. Three patients with severe recalcitrant uveitis with two to three previous pre-tocilizumab biological treatments had active uveitis throughout the follow-up. A recent retrospective study by Calvo-Río and colleagues reported a complete remission of severe uveitis that was refractory to conventional DMARDs and at least one anti-TNF agent in 19 of 25 patients after 12 months of tocilizumab treatment [36]. The first prospective study on tocilizumab efficacy in JIA-associated anti-TNF agent refractory uveitis is being conducted at present (the APTITUDE trial) [37].

There are several limitations to our study. As this was an observational study there were some missing data and the follow-up was shorter than 24 months for some patients. However, most of data regarding the JIA core set variables were available since they were collected prospectively to the JIA databases. In addition, the study population was rather small, 56 patients and $85.1 \mathrm{PYs}$, and included only one seropositive polyarthritis patient. It is therefore not possible to generalize our results to this or other missing categories of JIA. Nevertheless, this is one of the largest real-life studies reporting on the efficacy and safety of tocilizumab in pJIA patients.

In conclusion, our study showed that survival of tocilizumab was good and a large proportion of the hard-to-treat pJIA patients reached LDA at 12 months of treatment. The rate of LDA continued to increase among those remaining on tocilizumab throughout 24 months. Patients needed significantly fewer synthetic DMARDs, and systemic or intra-articular glucocorticoids during the 24-month followup. The rate of AES and SAEs was higher than that in previous register studies but lower than that of the original progenitor trial of tocilizumab in pJIA. 
Funding: This work was supported by Roche Oy. The study was investigator-initiated and Roche Oy had no role in the collection, analysis or interpretation of the data, the writing of the manuscript, or the decision to submit the manuscript for publication.

Disclosure statement: M.-M.G. has received a research grant from Roche for this study, and outside the submitted work, travel grants from Abbvie and Roche, and advisory board membership fees from Roche. T.R.-P. has received, outside the submitted work, travel grants from Abbvie, Pfizer and Roche. L.K. has received, outside the submitted work, travel grants from Abbvie, Merck Sharp \& Dohme (MSD) and Roche, advisory board membership fees from Roche, and speaker fees from Abbvie and Roche. K.M.-P. has received, outside the submitted work, travel grants from Abbvie, MSD, Pfizer and Roche. M.B. has received, outside the submitted work, travel grants from Abbvie and Roche, and speaker fees from Pfizer. A.P.-L. has received, outside the submitted work, a travel grant from Pfizer, and advisory board membership fees from Roche. K.A. has received, outside the submitted work, travel grants from Abbvie, MSD, Pfizer, Roche and Sobi, consultation fees from Novartis, Roche and Sobi, and advisory board membership fees from Roche. P.V. has received, outside the submitted work, travel grants from Abbvie, MSD, Pfizer, Roche and Sobi, speaker fees from Abbvie, MDS, Pfizer, Roche and Sobi, and advisory board membership fees from Roche.

\section{References}

1. Petty RE, Southwood TR, Manners P et al. . International League of Associations for Rheumatology classification of juvenile idiopathic arthritis: second revision, Edmonton, 2001. J Rheumatol 2004;31:390-2.

2. Ravelli A, Martini A. Juvenile idiopathic arthritis. Lancet 2007;369:767-78.

3. Nordal E, Zak M, Aalto $\mathrm{K}$ et al. . Ongoing disease activity and changing categories in a longterm nordic cohort study of juvenile idiopathic arthritis. Arthritis Rheum 2011;63:2809-18.

4. Wallace CA, Huang B, Bandeira M, Ravelli A, Giannini EH. Patterns of clinical remission in select categories of juvenile idiopathic arthritis. Arthritis Rheum 2005;52:3554-62.

5. Guzman J, Oen K, Tucker LB et al. . The outcomes of juvenile idiopathic arthritis in children managed with contemporary treatments: results from the ReACCh-Out cohort. Ann Rheum Dis 2015;74:1854-60.

6. Magnani A, Pistorio A, Magni-Manzoni S et al. . Achievement of a state of inactive disease at least once in the first 5 years predicts better outcome of patients with polyarticular juvenile idiopathic arthritis. J Rheumatol 2009;36:628-34.

7. Beukelman T, Patkar NM, Saag KG et al. . 2011 American College of Rheumatology recommendations for the treatment of juvenile idiopathic arthritis: initiation and safety monitoring of therapeutic agents for the treatment of arthritis and systemic features. Arthritis Care Res (Hoboken) 2011;63:465-82.

8. Ringold S, Weiss P, Colbert R et al. . Childhood Arthritis and Rheumatology Research Alliance consensus treatment plans for new-onset polyarticular juvenile idiopathic arthritis. Arthritis Care Res (Hoboken) 2014;66:1063-72.

9. Horneff G, Klein A, Ganser G et al. . Protocols on classification, monitoring and therapy in children's rheumatology (PRO-KIND): results of the working group Polyarticular juvenile idiopathic arthritis. Pediatr Rheumatol Online J 2017;15:78.

10. Otten MH, Anink J, Prince FHM et al. . Trends in prescription of biological agents and outcomes of juvenile idiopathic arthritis: results of the Dutch national Arthritis and Biologics in Children Register. Ann Rheum Dis 2015;74:1379-86.

11. Kearsley-Fleet L, Davies R, Baildam E et al. . Factors associated with choice of biologic among children with juvenile idiopathic arthritis: results from two UK paediatric biologic registers. Rheumatology (Oxford) 2016;55:1556-65.

12. Horneff $G$, Klein A, Klotsche $J$ et al. . Comparison of treatment response, remission rate and drug adherence in polyarticular juvenile idiopathic arthritis patients treated with etanercept, adalimumab or tocilizumab. Arthritis Res Ther 2016;18:272-81.

13. Amarilyo G, Tarp S, Foeldvari I et al. . Biological agents in polyarticular juvenile idiopathic arthritis: a meta-analysis of randomized withdrawal trials. Semin Arthritis Rheum 2016;46:3128.

14. Ringold S, Nigrovic PA, Feldman BM et al. . The Childhood Arthritis and Rheumatology Research Alliance consensus treatment plans: toward comparative effectiveness in the pediatric rheumatic diseases. Arthritis Rheum 2018;70:669-78. 
15. Berard R, Laxer R. Learning the hard way: clinical trials in juvenile idiopathic arthritis. Ann Rheum Dis 2018;77:1-2.

16. Horneff G, Ganser G, Foeldvari I et al. . Long term pharmacovigilance of biologics for juvenile idiopathic arthritis: the Biker-registry. Ann Rheum Dis 2018;77(Suppl 2):448-9.

17. Consolaro A, Ruperto N, Bazso A et al. . Development and validation of a composite disease activity score for juvenile idiopathic arthritis. Arthritis Rheum 2009;61:658-66.

18. Backström M, Tynjälä P, Ylijoki H et al. . Finding specific 10-joint Juvenile Arthritis Disease Activity Score (JADAS10) and clinical JADAS10 cut-off values for disease activity levels in nonsystemic juvenile idiopathic arthritis: a Finnish multicentre study. Rheumatology (Oxford) 2016;55:615-23.

19. Wallace C, Ruperto N, Giannini E, for the CARRA, PRINTO and PRCSG. Preliminary criteria for clinical remission for select categories of juvenile idiopathic arthritis. J Rheumatol 2004;31:2290-4.

20. Bethencourt Baute JJ, Sanchez-Piedra C, Ruiz-Montesinos D et al. .; on behalf of the BIOBADASER study group. Persistence and adverse events of biological treatment in adult patients with juvenile idiopathic arthritis: results from BIOBADASER. Arthritis Res Ther 2018;20:227-35.

21. Beukelman T, Anink J, Berntson L et al. . A survey of national and multi-national registries and cohort studies in juvenile idiopathic arthritis: challenges and opportunities. Pediatr Rheumatol Online J 2017;15:31-9.

22. Swart J, Giancane G, Horneff G et al. . for the PRINTO, BiKeR and the board of the Swedish Registry. Pharmacovigilance in juvenile idiopathic arthritis patients treated with biologic or synthetic drugs: combined data of more than 15,000 patients from Pharmachild and national registries. Arthritis Res Ther 2018;20:285-95.

23. Otten $\mathrm{MH}$, Prince $\mathrm{FH}$, Anink J et al. . Effectiveness and safety of a second and third biological agent after failing etanercept in juvenile idiopathic arthritis: results from the Dutch National ABC Register. Ann Rheum Dis 2013;72:721-7.

24. Bulatovic $M$, Heijstek $M$, Verkaaik $M$ et al. . High prevalence of methotrexate intolerance in juvenile idiopathic arthritis. Development and validation of a methotrexate intolerance severity score. Arthritis Rheum 2011;63:2007-13.

25. Brunner $\mathrm{HI}$, Ruperto N, Zuber Z et al. . Efficacy and safety of tocilizumab in patients with polyarticular-course juvenile idiopathic arthritis: results from a phase 3 , randomised, doubleblind withdrawal trial. Ann Rheum Dis 2015;74:1110-7.

26. Ravelli A, Consolaro A, Horneff $G$ et al. . Treating juvenile idiopathic arthritis to target: recommendations of an international task force. Ann Rheum Dis 2018;77:819-28.

27. Consolaro A, Bracciolini G, Ruperto $\mathrm{N}$ et al. . Remission, minimal disease activity, and acceptable symptom state in juvenile idiopathic arthritis: defining criteria based on the juvenile arthritis disease activity score. Arthritis Rheum 2012;64:2366-74.

28. Schmeling $\mathrm{H}$, Minden $\mathrm{K}$, Foeldvari I et al. . Efficacy and safety of adalimumab as the first and second biologic agent in juvenile idiopathic arthritis. The German Biologics JIA Registry. Arthritis Rheum 2014;66:2580-9.

29. Cabrera N, Lega JC, Kassai B et al. . Safety of biological agents in paediatric rheumatic diseases: a real-life multicenter retrospective study using the JIRcohorte database. Joint Bone Spine 2019;86:343-50.

30. De Benedetti F, Brunner $\mathrm{H}$, Ruperto $\mathrm{N}$ et al. . Randomized trial of tocilizumab in systemic juvenile idiopathic arthritis. N Engl J Med 2012;367:2385-95.

31. Tarkiainen $M$, Tynjälä $P$, Vähäsalo $P$, Lahdenne $P$. Occurrence of adverse events in patients with JIA receiving biologic agents: long-term follow-up in a real-life setting. Rheumatology (Oxford) 2015;54:1170-6.

32. Brunner H, Ruperto N, Zuber Z et al. . Efficacy and safety of tocilizumab in patients with polyarticular-course juvenile idiopathic arthritis: 2-year data from CHERISH. Arthritis Rheum 2014;66:S5-6.

33. Opoka-Winiarska V, uber Z, Alexeeva $\mathrm{E}$ et al. . Long-term, interventional, open-label extension study evaluating the safety of tocilizumab treatment in patients with polyarticular-course juvenile idiopathic arthritis from Poland and Russia who completed the global, international CHERISH trial. Clin Rheumatol 2018;37:1807-16.

34. Hashkes PJ, Uziel Y, Laxer RM. The safety profile of biologic therapies for juvenile idiopathic arthritis. Nat Rev Rheumatol 2010;6:561-71.

35. Pardeo M, Wang J, Ruperto $\mathrm{N}$ et al. .; for the PRINTO and PRCSG. Neutropenia during tocilizumab treatment is not associated with infection risk in systemic or polyarticular-course juvenile idiopathic arthritis. J Rheumatol 2019; doi:10.3899/jrheum.180795. 
36. Calvo-Río V, Santos-Gómez M, Calvo I et al. . Anti-Interleukin-6 receptor tocilizumab for severe juvenile idiopathic arthritis-associated uveitis refractory to anti-tumor necrosis factor therapy: a multicenter study of twenty-five patients. Arthritis Rheum 2017;69:668-75.

37. Ramanan AV, Dick AD, Jones AP et al. . A phase II trial protocol of tocilizumab in anti-TNF refractory patients with JIA-associated uveitis (the APTITUDE trial). BMC Rheumatol 2018;2:412. 


\section{Table 1}

Baseline patient and disease characteristics, total number of patients 56

Female

$40(71.4)$

JIA subtype

RF-negative polyarthritis

RF-positive polyarthritis

Extended oligoarthritis

History of uveitis

ANA positive

HLA-B27 positive

Age at disease onset, median (range), years

Disease duration at first DMARD start, median (IQR), days

Disease duration at first biological agent start, median (IQR), years

Disease duration at tocilizumab start, median (IQR), years

Disease activity parameters

Number of active joints, median (IQR)

$2.5(1-4)$

Number of joints with LOM, median (IQR)

$1.0(0-2)$

Physician global VAS, 0-100 scale, median (IQR)

$30.0(17-40)$

Patient's/parent's global VAS, 0-100 scale, median (IQR)

Patient's/parent's pain VAS, 0-100 scale, median (IQR)

ESR, median (IQR)

$8(5-13)$

CHAQ, median (IQR)

$0.625(0.125-1.0)$

JADAS-10, median (IQR)

$7.6(5.4-12.1)$

Concomitant DMARD treatment

MTX

Leflunomide

2 different DMARDs
$19(33.9)$

$11(19.6)$

47 (83.9)

$11(19.6)$ 
Data are reported as $n(\%)$ unless stated otherwise. ${ }^{a}$ Two patients had prednisolone alone. IQR: interquartile range; LOM: limitation of movement; VAS: visual analogue scale; CHAQ: Childhood Health Assessment Questionnaire-Disability Index; JADAS-10: 10-joint Juvenile Arthritis DAS. 
Table 2

Reasons for tocilizumab discontinuation

Number of patients (\%)

Inadequate treatment effect

$8(50)$

Inadequate treatment effect and adverse event

$6(37.5)$

Elevated transaminases

1

Elevated cholesterol values

1

Neutropenia

2

Itching

1

Restlessness and parents' request (patient had developmental disability) $\quad 1$

Adverse events

$2(12.5)$

Persistent diarrhoea, elevated transaminases

Obesity, fatty liver and elevated transaminases 
Table 3

The most common AEs and all SAEs

\begin{tabular}{|c|c|c|}
\hline & $\begin{array}{l}\text { Number } \\
\text { of AEs }\end{array}$ & $\begin{array}{l}\text { AEs/100 } \\
\text { PYs }\end{array}$ \\
\hline Any $A E$ & 171 & 200.9 \\
\hline Infections and infestations & 54 & 63.5 \\
\hline Upper respiratory infection & 29 & \\
\hline Acute otitis media & 8 & \\
\hline Skin infection & 6 & \\
\hline Gastroenteritis & 4 & \\
\hline Pharyngitis & 3 & \\
\hline Other infections & 4 & \\
\hline Gastrointestinal & 31 & 36.4 \\
\hline Nausea & 9 & \\
\hline Aphthous ulcers & 8 & \\
\hline Abdominal pain & 6 & \\
\hline Diarrhoea (non-infectious) & 5 & \\
\hline Dyspepsia & 3 & \\
\hline Blood and lymphatic system disorders & 22 & 25.9 \\
\hline Neutropenia & 22 & \\
\hline Investigations & 12 & 14.1 \\
\hline Elevated transaminases & 11 & \\
\hline Elevated LDL and cholesterol & 1 & \\
\hline Any SAE & 11 & 12.9 \\
\hline Infection & 3 & \\
\hline Infusion reaction requiring inpatient care & 1 & \\
\hline
\end{tabular}


Operation of avascular necrosis of hip

Surgical procedures (2 tympanostomy, 1 gastroscopy, 1 excision of ingrown toe nail) 4

Gastro- and colonoscopy due to persistent diarrhoea 1 AEs: adverse events; LDL: low-density lipoprotein; PY: patient year; SAEs: serious adverse event. 
Fig. 1 Use of biologics and reasons for their discontinuation before tocilizumab commencement
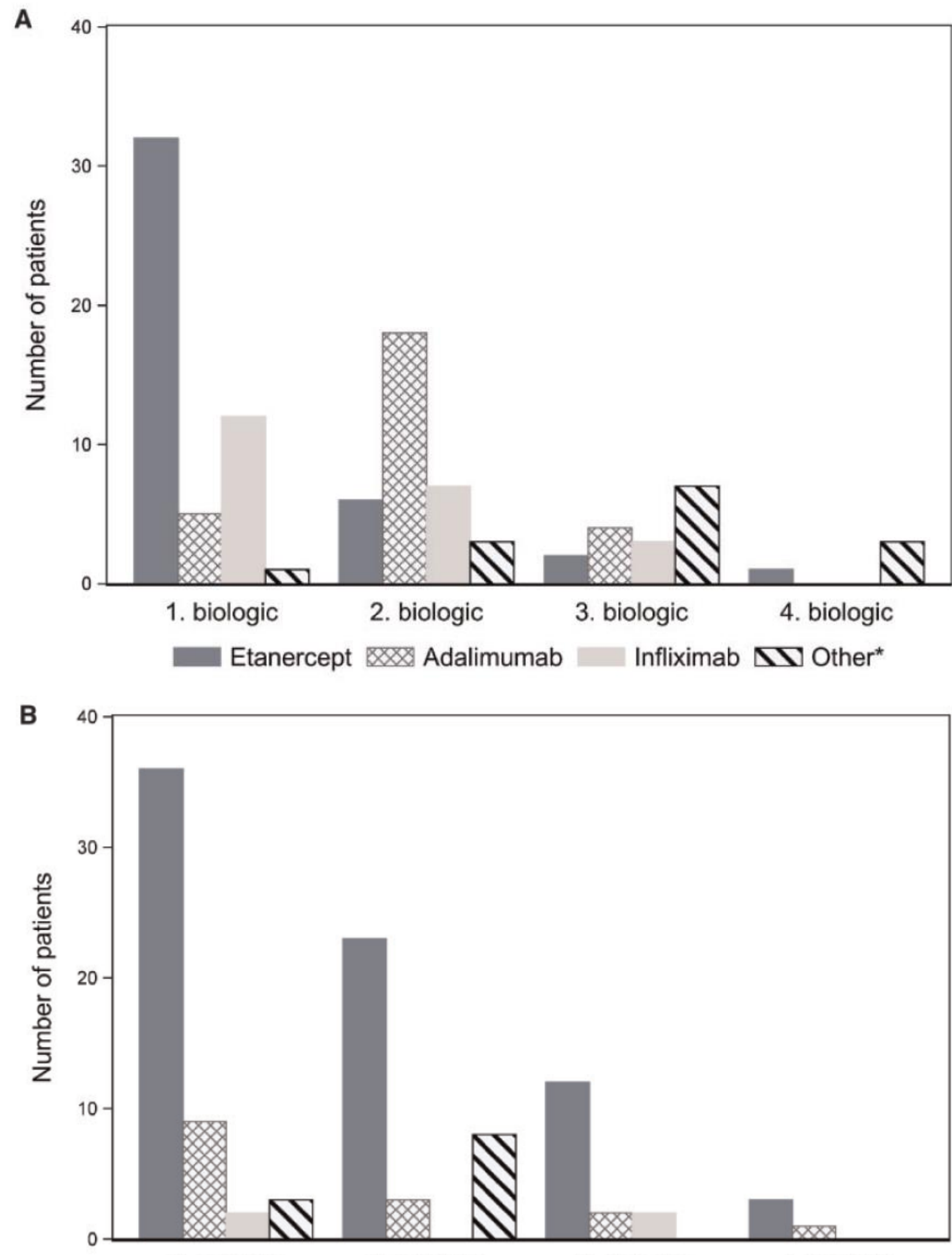

1. biologic

2. biologic

3. biologic

4. biologic

Inadequate treatment effect $x$ Adverse event Remission

$\Delta \nabla$ Anti-drug antibodies

(A) Distribution of different biological agents used before tocilizumab commencement in patients with polyarticular JIA. ${ }^{*}$ Other biological agents used; $1<$ sup $>$ st</sup $>$ biologic: one abatacept, $2<$ sup $>$ nd </sup> biologic: one abatacept and two golimumab, $3<$ sup $>$ rd $</$ sup $>$ biologic: five abatacept, one golimumab and one certolizumab pegol, $4<$ sup $>$ th $</$ sup $>$ biologic: 
three abatacept and one rituximab. (B) Reasons for discontinuation of biological agents before tocilizumab commencement in patients with polyarticular JIA. 
Fig. 2 Tocilizumab survival in patients with polyarticular JIA

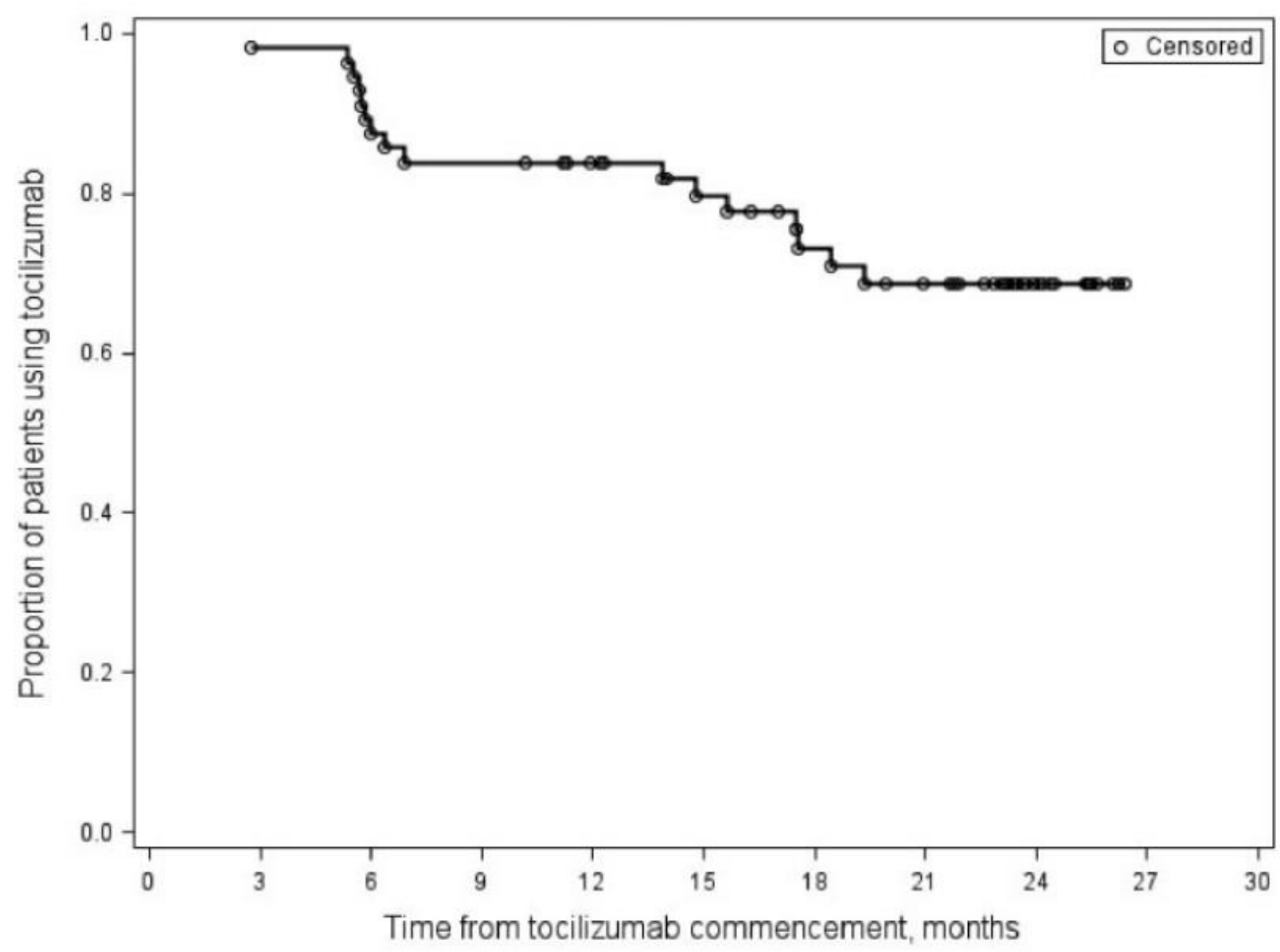


Fig. 3 Clinical outcomes of patients with polyarticular JIA remaining on tocilizumab treatment

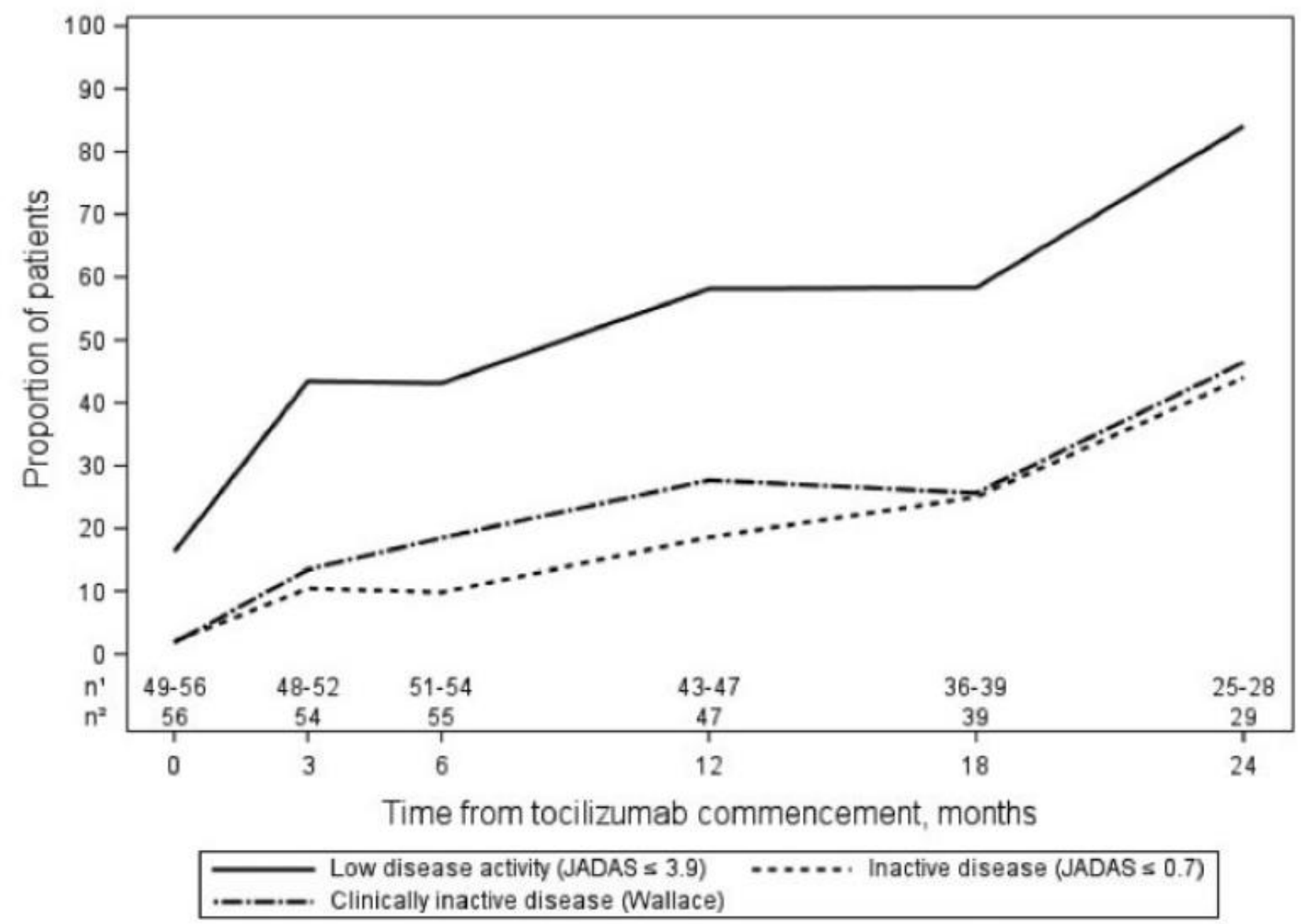

$\mathrm{n}^{1}=$ The numbers of patients with the data required for calculating JADAS values (the lower value) and assessing the clinically inactive disease by Wallace's criteria (the higher value) at each time point. $\mathrm{n}^{2}=$ The total numbers of patients remaining on tocilizumab treatment. JADAS: 10-joint Juvenile Arthritis DAS. 
